\title{
RELACIONES DEL AUTOCONCEPTO Y LA AUTOESTIMA CON LA SOCIABILIDAD, ESTABILIDAD EMOCIONAL Y RES- PONSABILIDAD EN ADOLESCENTES DE 14 A 17 AÑOS
}

\section{Maite Garalgordobil Alnhoa Durá}

Dpto. de Personalidad, Evaluación y Tratamientos Psicológicos. Facultad de Psicología. Universidad del País Vasco

\section{RESUMEN}

El estudio tiene tres objetivos: 1) analizar si existen diferencias en función del sexo en diversos parámetros de la personalidad durante la adolescencia; 2) estudiar las relaciones del autoconcepto y la autoestima con cooperación, habilidades sociales, sentimientos de felicidad, $y$ otras dimensiones de personalidad; $y$ 3) identificar variables predictoras de autoconcepto-autoestima. La muestra está constituida por 322 adolescentes de 14 a 17 años. El estudio utiliza una metodología descriptiva y correlacional. Para medir las variables se administran 6 instrumentos de evaluación. Los ANOVAs muestran puntuaciones significativamente superiores en los chicos en

\footnotetext{
Correspondencia: Maite Garaigordobil. Dpto. de Personalidad, Evaluación y Tratamientos Psicológicos. Facultad de Psicología. Universidad del Pals Vasco. Avda. de Tolosa 70. 20018 Donostia-San Sebastián.Tfno: 943-015936 / 943 - 015634 / Fax: 943 - 015670 . E-mail; maite.garaigordobil @ehu.es.

Nota. Maite Garaigordobil es profesora en el Dpto. de Personalidad, Evaluación y Tratamientos Psicológicos en la Facultad de Psicología de la Universidad del Pals Vasco y Ainhoa Durá miembro del equipo investigador. El estudio que se expone ha sido financiado por el Vicerrectorado de Investigación de la Universidad del País Vasco (1/UPV 00006.231-H-15910/ 2004). Correspondencia relacionada con el art/culo puede ser enviada a Maite Garaigordobil, Facultad de Psicología. Universidad del País Vasco, Avenida de Tolosa 70, 20018 Donostia-San Sebastián. E-rnail: maite.garaigordobil @ehu.es http://www.sc.ehu.es/garaigordobil.
} 
autoestima, asertividad inapropiada, sobreconfianza, desajuste emocional, y tolerancia al estrés, mientras que las chicas tienen superiores puntuaciones en cooperación, habilidades sociales apropiadas, adaptación a los cambios, disponibilidad hacia nuevas tareas y trabajo en equipo. Los coeficientes de Pearson sugieren que los adolescentes de ambos sexos con alto autoconcepto $y$ alta autoestima son cooperativos, tienen sentimientos de felicidad, tolerancia al estrés, inteligencia social, integración social, capacidad de trabajar en equipo, alta autoexigencia, tesón-constancia, baja asertividad inapropiada, baja impulsividad, pocos sentimientos de celos-soledad, bajo nivel de desajuste emocional, poca ansiedad y depresión, es decir, son estables emocionalmente, sociables y responsables.

Palabras clave: AUTOCONCEPTO, AUTOESTIMA, HABILIDADES SOCIALES, PERSONALIDAD, ADOLESCENCLA.

\section{SUMMARY}

The purpose of this study is threefold: 1) to assess possible gender differences in various parameters of personality during adolescence; 2) to study the concomitant relationships between self-concept/self-esteem, cooperation, social skills, feelings of happiness, and other personality traits; and 3) to identify the predictive variables of high self-concept and self-esteem. The sample consists of 322 adolescents aged 14 to 17 . The study uses descriptive and correlational methodology. Six evaluation Instruments were employed in the assessment of the variables. ANOVA results showed significantly higher scores in males in variables such as self-esteem, inappropriate assertiveness, overconfidence, emotional maladjustment, and stress tolerance, while females had significantly higher scores in cooperation, appropriate social skills, adaptation to change, attitude to new tasks, and capacity for teamwork. Pearson coefficients suggested that adolescents of both sexes with high self-concept/self-esteem are cooperative. In addition, they present feelings of happiness, tolerance to stress, capacity for social integration and teamwork, social intelligence, and perseverance, and tend to demand the 
best out of themselves. At the same time, they show low levels of inappropriate assertiveness, low levels of impulsiveness, few feelings of jealousy-loneliness, low levels of emotional maladjustment, and low levels of anxiety and depression. In sum, they are emotionally stable, sociable and responsible.

Key words: SELF-CONCEPT, SELF-ESTEEM, SOCLAL SKLLLS, PERSONALTT, ADOLESCENCE.

\section{AUTOCONCEPTO-AUTOESTIMA: DEFINICIÓN}

En los últimos años la investigación del autoconcepto y la autoestima está cobrando gran relevancia. La mayoría de los autores asocia el término autoconcepto a los aspectos cognitivos del conocimiento de uno mismo, y utilizan la denominación de autoestima para los aspectos evaluativo-afectivos. Recientemente, Cardenal y Fierro (2003) han definido el autoconcepto como un conjunto de juicios tanto descriptivos como evaluativos acerca de uno mismo; consideran que el autoconcepto expresa el modo en que la persona se representa, conoce y valora a ella misma, matizando que aunque a menudo se usan de manera equivalente autoconcepto y autoestima, ésta en rigor constituye el elemento valorativo del autoconcepto, del autoconocimiento.

\section{DIFERENCIAS DE GÉNERO EN VARIABLES DE LA PERSONA- LIDAD DURANTE LA ADOLESCENCIA}

Los resultados de los estudios que han analizado las diferencias de género en el autoconcepto y la autoestima muestran resultados divergentes. La mayor parte de las investigaciones encuentran diferencias significativas en la autoestima y observan menores puntuaciones en las chicas (Chabrol et al. 2004; Gracia, et al. 1999; Hagborg, 1993; Khanlou, 2004; Locker y Cropley, 2004; Robison et al. 2001; Walker, 2000), aunque en algunos estudios la magnitud de las diferencias es baja (Matud, Ibáñez, Marrero y Carballeira, 2003). Mientras que algunos trabajos encuentran diferencias entre sexos en el autoconcepto, observando en las mujeres peor autoconcepto global (Amezcua y Pichardo, 2000; Pastor, Balaguer y Garcla-Merita, 2003) y peor autoconcepto 
físico (Pastor et al. 2003; Klomsten, Skaalvik y Espnes, 2004), sin embargo, otros no han hallado diferencias significativas ni en el autoconcepto global de adolescentes de 12 a 14 años (Garaigordobil, Cruz y Pérez, 2003) ni en la autoestima (Lameiras y Rodríguez, 2003).

En relación a la conducta social, algunos estudios evidencian que las adolescentes tienen puntuaciones significativamente superiores en competencia social (Vordach, 2002), en habilidades sociales apropiadas (Méndez, Hidalgo e Inglés, 2002), en estrategias y conductas prosociales (Pakaslahti, Karjalainen y Keltikangas, 2002), así como en adaptación social (Clemente, Albiña y Domenech, 1998). Estudios transculturales ponen de relieve diferencias interculturales, ya que los adolescentes turcos tenían significativamente mayores niveles de asertividad que las chicas adolescentes, sin embargo, los suecos no evidenciaron diferencias de género en asertividad (Eskin, 2003). Por otro lado, algunos trabajos no han encontrado diferencias entre chicos y chicas en sentimientos de felicidad (Mahon, Yarcheski y Yarcheski, 2005), sin embargo otros estudios constatan que las chicas tienen más síntomas depresivos (Chabrol et al. 2004; Locker y Cropley, 2004) y de ansiedad (Locker y Cropley, 2004) que los chicos.

\section{AUTOCONCEPTO-AUTOESTIMA: RELACIONES CON LA CONDUCTA SOCIAL Y LA ESTABILIDAD EMOCIONAL}

Las investigaciones que estudian las relaciones entre el autoconcepto y la conducta prosocial han encontrado que los adolescentes prosociales tienen bajo autoconcepto negativo, alto autoconcepto positivo y alto autoconcepto-autoestima (Calvo, González y Martorell, 2001), alto autoconcepto académico, social y familiar (Gutiérrez y Clemente, 1993), y alta autoestima (Rigby y Slee, 1993). Los adolescentes que muestran consideración por los demás y sensibilidad social tienen alto autoconcepto social, y los que muestran conductas altruistas, tienen alto autoconcepto global (Garaigordobil et al. 2003). Son escasos los estudios correlacionales que han explorado las relaciones entre cooperación y autoconcepto durante la adolescencia, sin embargo, se ha confirmado que los programas de intervención para desarrollar la cooperación mejoran el autoconcepto (Garaigordobil, 1996, 1999ab, 2002, 2005; Garaigordobil y Echebarría, 
1995; Veenman, Kenter y Post, 2000). En esta dirección, Bauer (1997) observó que los adolescentes que empleaban más tiempo en actividades de aprendizaje cooperativo dentro del aula tenían mayor autoconcepto, y así mismo Hay, Ashman y Van Kraayenoord (1998) concluyeron que, desde la observación de los profesores, los alumnos con un alto autoconcepto se mostraban más cooperativos.

Además, varias investigaciones han encontrado relaciones positivas entre autoestima y habilidades sociales, asi como relaciones negativas con ansiedad social y soledad (Riggio, Throckmorton y De Paola, 1990). El estudio realizado por Bijstra, Bosma y Jackson (1994) halló relaciones positivas entre habilidades sociales e indicadores de funcionamiento psicosocial (autoestima, bienestar y apoyo social) en adolescentes de 13 a 15 años, mientras que Russomano (2000) encontró relaciones positivas entre autoconcepto y habilidades sociales en estudiantes de 8 a 11 años y sólo una tendencia en los estudiantes de 12 a 14 años. Otros trabajos han hallado relaciones positivas de la asertividad con autoestima (Deluty, 1981; Lefevre y West, 1981; Lorr y More, 1980) y con autoconcepto (Garaigordobil et al. 2003), identificando la autoestima social como predictor de asertividad (Arbanas, 1997). Algunas investigaciones ponen de relieve que los adolescentes con alta autoestima muestran pocas conductas de timidez (Lawrence y Bennett, 1992), que la adaptación social correlaciona con autoconcepto y es una variable predictora de éste, así como que un bajo nivel conductas sociales de ansiedad-timidez, pocos problemas de ansiedad y de retraimiento, han resultado ser variables predictoras de alto autoconcepto (Garaigordobil et al. 2003). Por otro lado, se han encontrado correlaciones negativas entre autoestima $e$ impulsividad (Heaven, 1991), entre autoestima y celos en chicas (Buunk, 1997), así como entre autoestima y soledad (Brage, Meredith y Woodward, 1993; Haines, Scalise y Ginter, 1993; Morol, 1987).

Estudios que han analizado las relaciones entre sentimientos de felicidady autoestima, han revelado que la autoestima es una variable predictora de felicidad, teniendo una relación negativa con depresión. La revisión de estudios clínicos y experimentales que llevó a cabo Coopersmith (1967) le condujeron a afirmar que las personas con alta autoestima eran más felices que los que tenían baja autoestima. Cheng y Furnham (2003) pudieron observar que la autoestima era un predictor 
de felicidad, y Neto (2001) encontró correlaciones positivas de la felicidad con autoestima, satisfacción con la vida, sociabilidad, así como correlaciones negativas con vergüenza, soledad, timidez, y ansiedad social. Complementariamente, Myers y Diener (1995) identificaron 4 rasgos que aparecían en las personas felices de todas edades: autoestima, sensación de control personal, optimismo y extraversión.

Por otro lado, se han explorado las relaciones del autoconceptoautoestima con rasgos asociados a la estabilidad emocional. Estudios con adolescentes han encontrado relaciones negativas entre autoconcepto y depresion (Alfeld y Sigelman, 1998; Fan y Fu, 2001; Garaigordobil, Durá y Pérez, 2005; Yang, 2002), así como entre autoestima y depresión (Garaigordobil et al. 2005; Hoffmann, Baldwin y Cerbone, 2003; Valentine, 2001). Otros trabajos demuestran que la alta autoestima es un factor protector de síntomas depresivos (Takakura y Sakihara, 2001), y que tanto el bajo autoconcepto (Braithwaite, 2004; Dowd, 2002) como la baja autoestima (Hong, 2002) son predictores de depresión. También se han confirmado relaciones negativas entre autoestima y ansiedad, sugiriendo que los adolescentes con alta autoestima muestran bajos niveles de ansiedad rasgo (Fickova, 1999; Newbegin y Owens, 1996; Yang, 2002). Finalmente, varios trabajos concluyen que las personas con alta autoestima son más constantes y persistentes (Di Paula y Capmbell, 2002; Mc Farlin, Baumeister y Blascovich, 1984).

\section{OBJETIVOS E HIPÓTESIS DEL ESTUDIO}

El estudio tiene 3 objetivos: 1) analizar si existen diferencias en función del sexo en diversos parámetros de la personalidad durante la adolescencia; 2) estudiar las relaciones del autoconcepto y la autoestima con cooperación, con habilidades sociales (habilidades sociales apropiadas, asertividad inapropiada, impulsividad, sobreconfianza, celos-soledad), con sentimientos de felicidad, y con 4 grandes dimensiones de personalidad o factores de primer orden como estabilidad emocional, apertura mental, sociabilidad y responsabilidad, a las que subyacen varias escalas o factores de segundo orden (desajuste emocional, ansiedad, depresión, tolerancia al estrés, tolerancia-flexibilidad ante diversas situaciones, adaptación a los 
cambios, interés por otras culturas, disponibilidad hacia nuevas tareas, inteligencia social, integración social, trabajo en equipo, autoexigencia profesional, dinamismo, tesón-constancia); y 3) Identificar variables predictoras del autoconcepto y la autoestima.

El estudio se plantea 5 hipótesis: 1) Se encontrarán diferencias significativas en el autoconcepto y la autoestima con puntuaciones superiores en los chicos; 2) Se hallarán diferencias significativas entre chicos y chicas, con puntuaciones superiores en las chicas en diversos tipos de conductas sociales positivas (cooperación, habilidades sociales apropiadas, capacidad para trabajar en equipo...), frente a los chicos que mostrarán puntuaciones significativamente más altas en asertividad inapropiada y en sobreconfianza; 3) No se darán diferencias entre chicos y chicas en sentimientos de felicidad, sin embargo, las chicas experimentarán más síntomas ansiosodepresivos, y los chicos mayor desajuste emocional; 4) los adolescentes de ambos sexos con alto autoconcepto y alta autoestima tendrán muchas conductas de cooperación, sentimientos de felicidad, alta tolerancia al estrés, inteligencia social, integración social, capacidad de trabajar en equipo, serán autoexigentes, constantes, manifestando baja asertividad inapropiada, baja impulsividad, pocos sentimientos de celos-soledad, bajo nivel de desajuste emocional, de ansiedad y de depresión, es decir, serán estables emocionalmente, sociables y responsables; y 5) Bajo nivel de depresión, muchos sentimientos de felicidad, habilidades sociales adecuadas y bajo nivel de impulsividad serán variables predictoras de autoestima y autoconcepto.

\section{MÉTODO}

\section{Participantes}

La muestra está constituida por 322 adolescentes de 14 a 17 años. El $42,8 \%$ tienen 14 años (138 sujetos), el 32,9\% (106 sujetos) 15 años, el $14,6 \%$ (47 sujetos) 16 años, y el $4 \%$ (13 sujetos) 17 años. La media de edad de la muestra es de 14,7 años. Los participantes se distribuyen en 16 grupos, inscritos en 4 centros escolares de la provincia de Guipúzcoa. Del conjunto de la muestra 9 grupos (193 
sujetos) cursan $3^{9}$ de Educación Secundaria Obligatoria y otros 7 grupos (129 sujetos) realizan $4^{2}$ curso. El $53,4 \%$ son chicos (172 sujetos) y el $45,3 \%$ (146 sujetos) chicas. En la muestra estudiada, 110 sujetos $(34,2 \%)$ cursan estudios en un centro educativo público, mientras que $212(65,8 \%)$ en centros educativos privados. En lo que al nivel socio-económico y cultural se refiere, se les preguntó el nivel de estudios tanto del padre como de la madre. Casi una cuarta parte de la muestra indica que el padre ha estudiado hasta los 14 años (23 $\%)$ o son licenciados $(23,9 \%)$, mientras casi una cuarta parte de las madres $(23,9 \%)$ son diplomadas o licenciadas $(20,5 \%)$. La mayoría de los padres $(90,4 \%)$ y de las madres $(80,4 \%)$ trabaja. A todos los participantes se les informó de los objetivos del trabajo así como de la voluntariedad para participar en el mismo, y se les solicitó el consentimiento informado.

\section{Diseño y procedimiento}

El estudio utiliza una metodología descriptiva y correlacional, buscando establecer relaciones de concomitancia del autoconceptoautoestima con cooperación, habilidades sociales, sentimientos de felicidad y 4 dimensiones de personalidad: estabilidad emocional, apertura mental, sociabilidad y responsabilidad. Con la finalidad de medir las variables dependientes se administran 6 instrumentos de evaluación con adecuadas garantías psicométricas de fiabilidad y validez. La batería de evaluación la implementan los psicólogos de los centros educativos con la colaboración de una licenciada en psicología en el transcurso de 3 sesiones de evaluación.

\section{Instrumentos de evaluaclón}

Con la finalidad de medir las variables dependientes (autoconcepto, autoestima, habilidades sociales, cooperación, sentimientos de felicidad, sociabilidad, estabilidad emocional, apertura mental, responsabilidad) se administran 6 instrumentos de evaluación.

LAEA. Listado de adjetivos para la evaluación del autoconcepto en adolescentes $y$ adultos (Garaigordobil, en prensa). El listado está compuesto por 57 adjetivos y se solicita al sujeto que valore en una 
escala de estimación de 0 a 4 (nada-mucho) en qué grado estos adjetivos le definen o describen su personalidad. Un estudio realizado con una muestra de 634 sujetos obtuvo un alpha de Cronbrach de 0,92 , y un coeficiente de Spearman-Brown de 0,83. La fiabilidad testretest obtenida con una muestra de 142 estudiantes universitarios y un intervalo de 40 dias fue alta $(r=0,83)$, evidenciando la estabilidad temporal de la prueba. Para analizar la validez del LAEA, se realizaron correlaciones con otros instrumentos que miden el autoconcepto (AF5, Garcia y Musitu, 1999) obteniéndose correlaciones significativas positivas $(r=0,71)$ que muestran una validez de constructo o concurrente aceptable. Estudios de validez discriminante del LAEA con una muestra de 1.578 sujetos de 12 a 65 han confirmado las relaciones negativas entre el LAEA y síntomas psicopatológicos medidos con el SCL-90-R (Derogatis, 2002).

EA. Escala de autoestima (Rosenberg, 1965). Esta escala evalúa la autoestima general con 10 afirmaciones que aluden a sentimientos globales de autovaloración (en general estoy satisfecho conmigo mismo), 5 de los cuales están redactados en sentido positivo y 5 en sentido negativo. El sujeto debe leer las afirmaciones e informar en qué medida pueden ser aplicadas a él mismo, haciendo la valoración sobre una escala de tipo Likert con 4 categorias de respuesta (desde muy de acuerdo, a muy en desacuerdo). La fiabilidad de la prueba ha sido ampliamente documentada en la literatura. McCarthy y Hoge (1982) han informado de coeficientes de consistencia (alpha de Cronbach) que se sitúan entre 0,74 y 0,77 , y de fiabilidad test-retest de 0,63 (intervalo de 7 meses) y de 0,85 (intervalo de 2 semanas). La validez de la escala como medida unidimensional de la autoestima ha sido también cornprobada en varios estudios (Rosenberg, 1965; Silber y Tippett, 1965).

EC. Escala de cooperatividad (Rigby, Cox y Black, 1997). Con 18 ítems la escala mide el grado de cooperación definido como "la manera de actuar de forma conjunta y coordinada, en el trabajo, en el ocio o en relaciones sociales, en la búsqueda o compartimiento de objetivos, en el placer de compartir actividades o simplemente para promover las relaciones". Las respuestas se dan en una escala Likert de 5 puntos teniendo en cuenta que 1 es totalmente desacuerdo y 5 totalmente de acuerdo. De los 18 ítems que posee la prueba, 9 
de estos Items reflejan actitudes cooperativas y 9 actitudes no cooperativas. En un estudio llevado a cabo en Australia con una muestra de adolescentes, se obtuvo una fiabilidad adecuada $(a ́=0,77)$. En el estudio original se investigó la validez concurrente, y los resultados obtenidos fueron que las correlaciones realizadas con el número de amigos fueron bajas $(r<0,20)$, sugiriendo que la calidad de los amigos es mejor indicador de cooperación que la cantidad de amigos, mostrando correlaciones significativas con sentimientos de felicidad.

MESSY. Escala de evaluación de habilidades sociales en los niños (Trianes et al. 2002). Este instrumento está compuesto por 62 items, presentando la opción de respuesta en una escala Likert del 1 "nunca" al 4 "siempre". La escala permite evaluar 5 factores: habilidades sociales apropiadas (conductas como expresividad emocional, tener amigos, compartir), asertividad inapropiada (conductas agresivas, de burla o abuso de los demás), impulsividad (conductas como enfadarse fácilmente o interrumpir a los demás), sobreconfianza (conductas de sobrevaloración del Yo) y celos-soledad (sentimientos de soledad, ausencia de amigos). Los estudios realizados con la versión castellana del MESSY en una muestra de 634 adolescentes de entre 12 y 17 años, mostraron una consistencia interna alta (alpha $=0,88$ ). En otro estudio se calculó el coeficiente de correlación de Pearson con la puntuación total de la MESSY y las puntuaciones de la ASA (Assertiveness Scale for Adolescents), del TISS (Teenage Inventory of Social Skills), y del ASCA (Assertiveness Scale for Children and Adolescents). Las conductas sociales inapropiadas correlacionaron negativamente con asertividad y conducta prosocial y, positivamente con agresividad y conducta asocial, siendo la correlación con sumisión negativa.

CF. El cuestionario de felicidad de Oxford (Hills y Argyle, 2002). Esta escala se deriva del Oxford Happiness Inventory $(\mathrm{OHI})$, que reducida a 29 ftems trata de medir la felicidad de carácter general de cada individuo, es decir, el bienestar psicológico. El sujeto expresará su grado de acuerdo con una escala likert de 1 a 6 en cada enunciado, siendo 1 totalmente en desacuerdo hasta 6 totalmente de acuerdo. Los estudios realizados de esta escala con una muestra de sujetos entre 13 y 68 años verifican una buena fiabilidad $(\hat{a}=0,91)$. La validez del constructo el Oxford Happiness Inventory fue evaluada con asociaciones de diferentes medidas de diferencias individuales 
de rasgos y variables cognitivas. Así, en el estudio original se compararon las asociaciones del $\mathrm{OHI}$ y del $\mathrm{OHQ}$. En términos de validez de constructo, el OHQ mostró ser la medida más preferente.

TPT. Test de personalidad de TEA. (Corral, Pamos, Pereña, y Seisdedos, 2002). La prueba presenta 160 afirmaciones frente a las que el adolescente debe informar la frecuencia con la que puede autoaplicarse su contenido "casi nunca, a veces, con trecuencia o casi siempre". El test permite explorar 4 grandes dimensiones de personalidad: estabilidad emocional, apertura mental, sociabilidad y responsabilidad. Complementariamente informa de un conjunto de factores de segundo orden subyacentes a estas dimensiones como: 1) desajuste asociado a inestabilidad, a alteración fácil ante las demandas del entorno y sus emociones; 2) ansiedad relacionada con tensión, sobreexcitación, preocupación; 3) depresión vinculada a preocupaciones, creencias irracionales, pobre valoración de sí mismo; 4) tolerancia al estrés asociada a dificultades para enfrentarse con serenidad a las situaciones de tensión; 5) tolerancia y flexibilidad ante diversas situaciones; 6) adaptación a los cambios o las situaciones novedosas; 7) interés por otras culturas en relación a la profundización del conocimiento de las mismas; 8) disponibilidad hacia nuevas tareas; 9) inteligencia social para crear relaciones apropiadas con otros; 10) integración social basada en el respeto y aceptación de normas y tradiciones sociales; 11 ) trabajo en equipo definido en términos de participación y colaboración; 12) autoexigencia profesional que evalúa la motivación y ambición profesional; 13) dinamismo o energía para trabajar; y 14) tesón-constancia que evalúa la tenacidad y capacidad para perseverar en una tarea incluso ante condiciones adversas. Los coeficientes alpha, que oscilan entre 0,40 y 0,80 , pueden ser considerados suficientemente elevados para un cuestionario de personalidad y con unas escalas con pocos elementos (10 por escala). En relación a la validez se informa sobre aquellos elementos de cada escala que tienen la mayor saturación. Para ello se han entresacado de cada escala aquel elemento, con redacción positiva hacia la variable, que presenta la correlación más elevada con dicha escala y el elemento que, con redacción contraria al contenido de la escala, tiene mayor correlación con ella. Como medida del grado de saturación en la escala se calculó el IHc (índice de 
homogeneidad corregida) obteniendo índices de homogeneidad adecuados para cada rasgo.

\section{RESULTADOS}

Autoconcepto, autoestima, cooperación, habilidades sociales, sentimientos de felicidad $y$ otros rasgos de personalidad: Diferencias en función del género

Se realizó un ANOVA para explorar la existencia de diferencias significativas en función del sexo en todas las variables objeto de estudio. Como se puede observar en la Tabla 1, los resultados muestran diferencias significativas en función del género en autoestima con puntuaciones superiores en los chicos $(M=31,65)$ frente a las chicas $(M=28,21)$. En relación a variables relacionadas con la conducta social, por ejemplo, en cooperación las chicas tienen mayores puntuaciones $(M=71,46)$ que los chicos $(M=67,59)$, en habilidades sociales apropiadas también las chicas muestran puntuaciones superiores $(M=71,82)$ que los chicos $(M=69,32)$, mientras que éstos manifiestan superiores puntuaciones en asertividad inapropiada $(M=$ $28,68)$, que las chicas ( $M=24,01)$, así como en sobreconfianza en la que los chicos muestran mayor puntuación $(M=9,13)$ que las chicas $(M=7,82)$. En lo que se refiere a rasgos de personalidad, como se puede observar en la Tabla 1, los chicos tienen puntuaciones significativamente superiores en desajuste emocional $(M=9,58)$, y en tolerancia al estrés $(M=14,93)$, mientras que las chicas muestran inferiores puntuaciones en estas variables respectivamente $(M=8,46$; $M=13,07)$. Sin embargo, las chicas tienen puntuaciones significativamente mayores en ansiedad $(M=13,78)$ y depresión ( $M$ $=13,36)$, que los chicos $(M=12,12 ; M=11,99)$. Complementariamente, las adolescentes tienen significativamente superiores puntuaciones en adaptación a los cambios $(M=16,55)$, y en disponibilidad hacia nuevas tareas $(M=18,86)$, que los adolescentes $(M=15,74 ; M=$ $16,26)$. Además, se han encontrado diferencias significativas en trabajo en equipo con mayores puntuaciones en las chicas $(M=18,90)$, que los chicos $(M=16,91)$. Los resultados del análisis descriptivo evidencian que los adolescentes tienen puntuaciones significativamente 
superiores en autoestima, asertividad inapropiada, sobreconfianza, desajuste emocional y tolerancia al estrés, mientras que las adolescentes muestran superiores puntuaciones en cooperación, habilidades sociales apropiadas, adaptación a los cambios, disponibilidad hacia nuevas tareas, capacidad para trabajar en equipo, ansiedad y depresión.

Tabla 1. Medias, desviaciones tipicas y resultados del análisis de varianza para chicos y chicas en autoconcepto, autoestima, cooperación, habilidades sociales, sentimientos de felicidad y rasgos de personalidad

\begin{tabular}{|c|c|c|c|c|c|}
\hline & \multicolumn{2}{|c|}{ Chicos $(n=172)$} & \multicolumn{2}{|c|}{ Chicas $(n=146)$} & \multirow{2}{*}{$\begin{array}{c}\text { Anova } \\
F(1,316)\end{array}$} \\
\hline & $M$ & $\overline{D T}$ & $M$ & $\overline{D T}$ & \\
\hline EA Autoestima & 31,65 & 4,64 & 28,21 & 5,47 & $35,86^{* * *}$ \\
\hline LAEA Autoconcepto & 145,48 & 30,76 & 145,09 & 25,55 & 0,01 \\
\hline EC Cooperación & 67,59 & 10,85 & 71,46 & 7,83 & $12,10^{* * *}$ \\
\hline MESSY Habilidades sociales apropiadas & $69, \overline{32}$ & 9,95 & 71,82 & 7,87 & $5,92 *$ \\
\hline MESSY Asertividad inapropiada & 28,68 & 7,80 & 24,01 & 4,41 & $40,47 * * *$ \\
\hline MESSY Impulsividad & 9,31 & 2,39 & 9,49 & 2,00 & 0,50 \\
\hline MESSY Sobreconfianza & 9,13 & 2,55 & 7,82 & 1,62 & $28,09 * * *$ \\
\hline MESSY Celos-Soledad & 10,90 & 3,15 & 11,24 & 2,73 & 1,05 \\
\hline CF Felicidad & 122,42 & 18,25 & 120,84 & 19,21 & 0,53 \\
\hline TPT Desajuste emocional & 9,58 & 4,33 & 8,46 & 3,76 & $5,69 *$ \\
\hline TPT Ansiedad & 12,12 & 3,41 & 13,78 & 3,86 & $15,81 * * *$ \\
\hline TPT Depresión & 11,99 & 4,08 & 13,36 & 4,83 & $7,21 * *$ \\
\hline TPT Tolerancia estrés & 14,93 & 3,23 & 13,07 & 3,80 & $21,42 * * *$ \\
\hline TPT Tolerancia - Flexibilidad & 16,23 & 3,36 & 16,84 & 3,64 & 2,28 \\
\hline TPT Adaptación a los cambios & 15,74 & 2,81 & 16,55 & 3,66 & $4,75^{*}$ \\
\hline TPT Interés por otras culturas & 13,60 & 5,20 & 14,50 & 5,17 & 2,33 \\
\hline TPT Disponibilidad hacia nuevas tareas & 16,26 & 3,39 & 18,86 & 3,42 & $44,23 * * *$ \\
\hline TPT Inteligencia social & 15,40 & 3,28 & 15,48 & 3,48 & 0,04 \\
\hline TPT Integración social & 17,36 & 3,60 & 17,86 & 3,61 & 1,44 \\
\hline TPT Trabajo en equipo & 16,91 & 3,76 & 18,90 & 3,29 & $23,66 * * *$ \\
\hline TPT Autoexigencia profesional & 14,38 & 4,12 & 14,21 & 4,20 & 0,12 \\
\hline TPT Dinamismo & 15,18 & 3,49 & 14,83 & 2,98 & 0,88 \\
\hline TPT Tesón - constancia & 16,50 & 3,34 & 16,98 & 3,87 & 1,36 \\
\hline
\end{tabular}


Relaciones del autoconcepto y la autoestima con cooperación, habilidades sociales, sentimlentos de felicldad $y$ otros rasgos de personalidad

Para explorar las relaciones entre autoestima y autoconcepto con el resto de las variables objeto de estudio se calcularon los coeficientes de correlación de Pearson con las puntuaciones obtenidas en la EA y el LAEA con la EC, la MESSY, el CF, y el TPT, cuyos resultados pueden observarse en la Tabla 2.

Como se puede observar en la Tabla 2, se encuentran relaciones positivas significativas entre autoestima y cooperación, habilidades sociales apropiadas (en chicos), sentimientos de felicidad, tolerancia al estrés, tolerancia-flexibilidad (en chicos), adaptación a los cambios (en chicos), inteligencia social, integración social, trabajo en equipo, autoexigencia profesional, dinamismo (en chicos), y tesón-constancia. Además, se encuentran relaciones negativas significativas entre autoestima y asertividad inapropiada, impulsividad, celos-soledad, desajuste emocional, ansiedad, y depresión. Globalmente se han encontrado correlaciones positivas de la autoestima con estabilidad emocional, sociabilidad y con responsabilidad, así como relaciones negativas con apertura mental.

Por consiguiente, los resultados sugieren que los adolescentes, de ambos sexos, de 14 a 17 años, con altas puntuaciones en autoestima tienen con mayor probabilidad muchas conductas cooperativas, muchas habilidades sociales apropiadas (únicamente en chicos), sentimientos de felicidad, alta tolerancia al estrés, mucha toleranciaflexibilidad (únicamente en chicos), alta capacidad de adaptación a los cambios (únicamente en chicos), alta inteligencia social, integración social y alta capacidad para trabajar en equipo, manifestando alta autoexigencia profesional, dinamismo (únicamente en chicos), y alto nivel de tesón-constancia. Además, los adolescentes de ambos sexos con altas puntuaciones en autoestima muestran, con mayor probabilidad, baja asertividad inapropiada, baja impulsividad, pocos celos y sentimientos de soledad, bajo nivel de desajuste emocional así como poca ansiedad o depresión. Globalmente, los adolescentes con altas puntuaciones en autoestima tienen mayor probabilidad de ser estables emocionalmente, sociables y responsables. 
Tabla 2. Coeficientes de correlación de Pearson del autoconceptoautoestima con cooperación, habilidades sociales, felicidad y rasgos de personalidad on chicos $y$ chicas

\begin{tabular}{|c|c|c|c|c|}
\hline & \multicolumn{2}{|c|}{$\begin{array}{c}\text { EA } \\
\text { Autoestima }\end{array}$} & \multicolumn{2}{|c|}{$\begin{array}{c}\text { LAEA } \\
\text { Autoconcepto }\end{array}$} \\
\hline & $\begin{array}{c}\text { Chicos } \\
(n=172)\end{array}$ & $\begin{array}{c}\text { Chicas } \\
(n=146)\end{array}$ & $\begin{array}{c}\text { Chicos } \\
(n=172)\end{array}$ & $\begin{array}{c}\text { Chicas } \\
(n=146)\end{array}$ \\
\hline EC Cooperación & $0,31 * * *$ & $0,32^{* * * *}$ & $0,46^{* * * *}$ & $0,34 * * *$ \\
\hline MESSY Habilidades sociales apropiadas & $0,24^{* *}$ & 0,08 & $0,61^{* * *}$ & $0,45^{* * *}$ \\
\hline MESSY Asertividad inapropiada & $-0,22^{* *}$ & $-0,26 * * *$ & $-0,36 * * *$ & $-0,22 *$ \\
\hline MESSY Impulsividad & $-0,37 * * *$ & $-0,30 * * *$ & $-0,38 * * *$ & $-0,18 *$ \\
\hline MESSY Sobreconfianza & $-0,11$ & 0,06 & $-0,15$ & 0,11 \\
\hline MESSY Celos-Soledad & $-0,48 * * *$ & $-0,53^{* * *}$ & $-0,36 * * *$ & $-0,44^{* * *}$ \\
\hline CF Felicidad & $0,50^{* * *}$ & $0,63 * * *$ & $0,60^{* * *}$ & $0,63^{* * *}$ \\
\hline TPT Desajuste emocional & $-0,28 * * *$ & $-0,36 * * *$ & $-0,34 * * *$ & $-0,30 * * *$ \\
\hline TPT Ansiedad & $-0,29 * * *$ & $-0,51 * * *$ & $-0,27^{* *}$ & $-0,45 * * *$ \\
\hline TPT Depresión & $-0,50 * * *$ & $-0,64 * * *$ & $-0,53 * * *$ & $-0,49 * * *$ \\
\hline TPT Tolerancia estrés & $0,32 * * *$ & $0,40 * * *$ & $0,41 * * *$ & $0,32 * * *$ \\
\hline TPT Toleran-Flexibilidad & $0,18^{*}$ & 0,02 & 0,12 & $-0,60$ \\
\hline TPT Adaptación a los cambios & $0,27^{* * *}$ & 0,04 & 0,09 & 0,04 \\
\hline TPT Interés por otras culturas & 0,12 & 0,09 & $0,26 * *$ & 0.12 \\
\hline TPT Disponibilidad hacia nuevas tareas & 0,09 & $-0,13$ & $0,32 * * *$ & 0,04 \\
\hline TPT Inteligencia social & $0,37^{* * *}$ & $0,40 * * *$ & $0,42^{* * *}$ & $0,41^{* * *}$ \\
\hline TPT Integración social & $0,38^{* * *}$ & $0,37 * * *$ & $0,43 * *$ & $0,31 * * *$ \\
\hline TPT Trabajo en equipo & $0,28^{* * *}$ & $0,23^{* *}$ & $0,39 * * *$ & $0,32^{* * *}$ \\
\hline TPT Autoexigencia profesional & $0,49^{* * * *}$ & $0,42^{* * * *}$ & $0,51^{* * *}$ & $0,46^{* * *}$ \\
\hline TPT Dinamismo & $0,19 *$ & 0,15 & $0,46^{* * *}$ & $0,37^{* * *}$ \\
\hline TPT Tesón - constancia & $0,27^{* * *}$ & $0,42^{* * *}$ & $0,32 * * *$ & $0,34^{* * *}$ \\
\hline TPT Estabilidad Emocional & $0,40 * * *$ & $0,51^{* * *}$ & $0,46^{* * *}$ & $0,45^{* * *}$ \\
\hline TPT Apertura mental & $-0,22 * *$ & $-0,20^{*}$ & $-0,35^{* * *}$ & $-0,26^{* *}$ \\
\hline TPT Sociabilidad & $0,29 * * *$ & $0,31^{* * *}$ & $0,40^{* * *}$ & $0,43^{* * *}$ \\
\hline TPT Responsabilidad & $0,42^{* * *}$ & $0,44^{* * *}$ & $0,48^{* * *}$ & $0,43^{* * *}$ \\
\hline
\end{tabular}

${ }^{*} p<0,05 \quad * * p<0,011^{* * *} p<0,001$ 
Por otro lado (ver Tabla 2), se encuentran relaciones positivas significativas entre autoconcepto y cooperación, habilidades sociales apropiadas, sentimientos de felicidad, tolerancia al estrés, interés por otras culturas (en chicos), disponibilidad hacia nuevas tareas (en chicos), inteligencia social, integración social, trabajo en equipo, autoexigencia profesional, dinamismo y tesón-constancia. Además, se encuentran relaciones negativas significativas entre autoconcepto y asertividad inapropiada, impulsividad, celos-soledad, desajuste emocional, ansiedad y depresión. Globalmente se han encontrado correlaciones positivas del autoconcepto con la estabilidad emocional, sociabilidad y responsabilidad, así como negativas con apertura mental.

Por consiguiente, los resultados sugieren que los adolescentes de 14 a 17 años, de ambos sexos, con altas puntuaciones en autoconcepto tienen con mayor probabilidad muchas conductas cooperativas, habilidades sociales apropiadas, sentimientos de felicidad, tolerancia al estrés, alto interés por otras culturas (únicamente en chicos), disponibilidad hacia nuevas tareas (únicamente en chicos), alto nivel de inteligencia social, de integración social y alta capacidad de trabajo en equipo, mostrando alto nivel de autoexigencia, dinamismo y tesón-constancia. Además, los adolescentes con altas puntuaciones en autoconcepto, con mayor probabilidad, manifiestan baja asertividad inapropiada, baja impulsividad, pocos celos y sentimientos soledad, bajo nivel de desajuste emocional, baja ansiedad y baja depresión. Globalmente, los adolescentes con altas puntuaciones en autoconcepto, con mayor probabilidad, son estables emocionalmente, sociables y responsables.

En síntesis, los resultados de los análisis correlacionales sugieren que los adolescentes de ambos sexos con altas puntuaciones en autoconcepto y autoestima, con mayor probabilidad: 1) son cooperativos, es decir, son capaces de actuar de forma conjunta y coordinada, en el trabajo, en el ocio o en relaciones sociales...; 2) tienen sentimientos de felicidad, de bienestar psicológico; 3) manifiestan alta tolerancia al estrés, siendo capaces de enfrentarse con serenidad a situaciones de tensión; 4) tienen alto nivel de inteligencia social, es decir, son capaces de crear relaciones apropiadas con otros; 5) manifiestan alto nivel de integración social, basada en el respeto y la aceptación de normas y tradiciones sociales; 
6) disponen de alta capacidad de trabajar en equipo, para participar y colaborar; 7) son autoexigentes, con motivación y ambición profesional; 8) tienen tesón, constancia, tenacidad, capacidad para perseverar en una tarea incluso ante condiciones adversas; 9) manifiestan baja asertividad inapropiada, es decir, pocas conductas agresivas, de burla o abuso de los demás; 10) tienen baja impulsividad en las interacciones sociales, pocas conductas de enfadarse fácilmente o interrumpir a los demás; 11) experimentan pocos sentimientos de celos y de soledad; 12) manifiestan un bajo nivel de desajuste emocional, es decir, son estables y no se alteran fácilmente ante las demandas del entorno; 13) tienen bajos niveles de ansiedad relacionada con tensión, sobreexcitación, preocupación; y 14) muestran bajos niveles de depresión vinculada a preocupaciones, creencias irracionales, pobre valoración de sí mismo... Así, los adolescentes con altas puntuaciones en autoconcepto y autoestima, con mayor probabilidad, son estables emocionalmente, sociables y responsables.

Finalmente, se ha calculado la potencia de las pruebas de correlación realizadas, en las variables en las que se han evidenciado correlaciones significativas $(p<0,05)$ mediante el GPOWER (2.0 Faul y Erdfelder, 1992). Las correlaciones expresadas en autoestima (chicos) muestran elevados niveles de potencia por encima de 0,90 en todas las variables, excepto en tolerancia-flexibilidad $(0,77)$ y dinamismo $(0,81)$ cuyos resultados muestran un nivel de potencia algo inferior. Las correlaciones en autoestima (chicas) muestran también elevados niveles de potencia por encima de .94, con la excepción de apertura mental $(0,79)$ y trabajo en equipo $(0,88)$. En autoconcepto, la potencia de las correlaciones en los chicos es superior a 0,96 en todas las variables, $y$ en las chicas es superior a 0,94 para todas las variables con la excepción de asertividad inapropiada $(0,85)$ e impulsividad $(0,71)$. Por consiguiente, la asunción de que las variables tienen las relaciones encontradas puede afirmarse.

\section{Variables predictoras de autoconcepto y autoestima}

Para identificar las variables que predicen una alta puntuación en autoconcepto y autoestima se realizó un análisis de regresión lineal múltiple, paso a paso, cuyos resultados se presentan en la Tabla 3. 


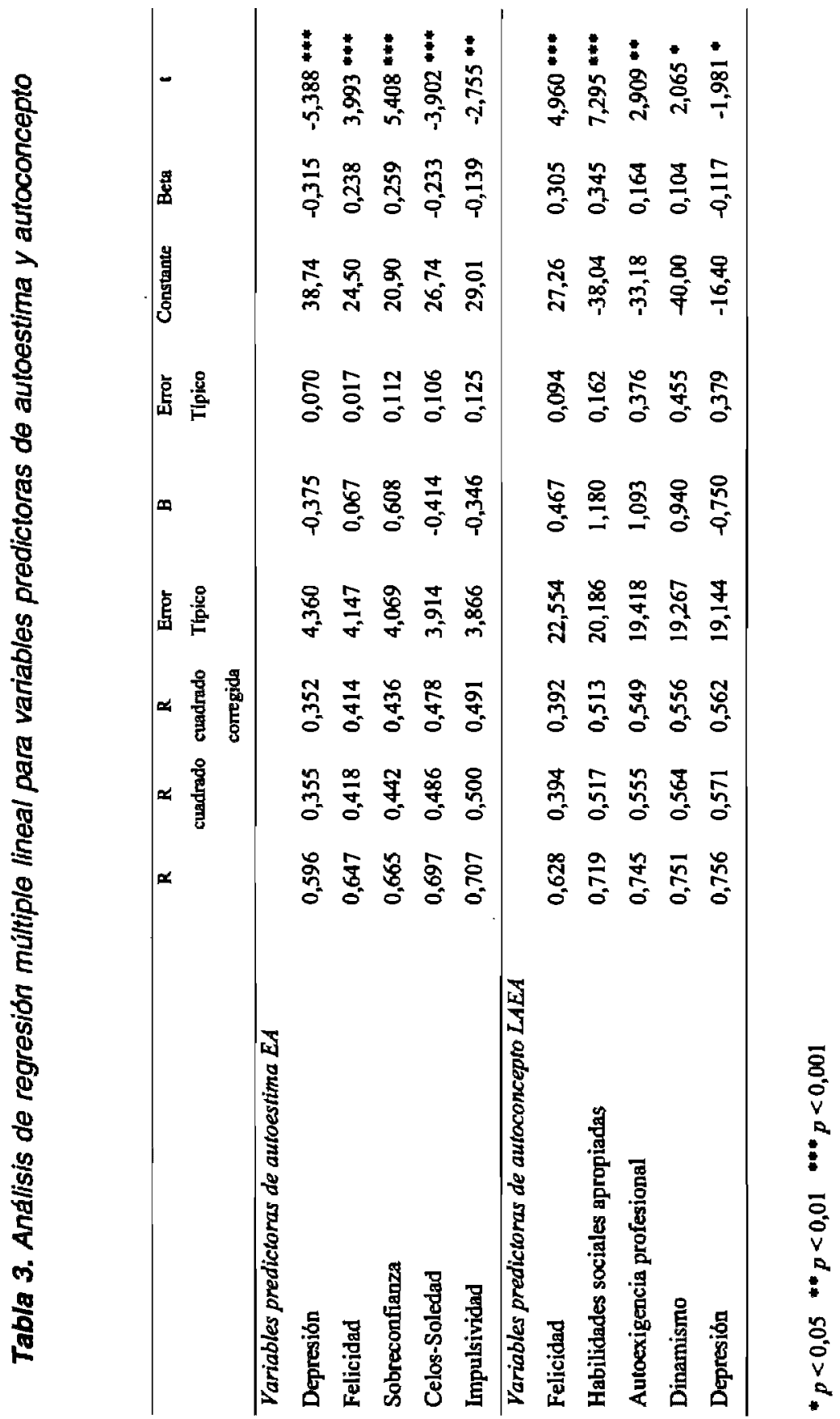


Como se puede observar en la Tabla 3, del conjunto de las variables predictoras de autoestima, 5 resultaron estadísticamente significativas: depresión (beta $=-0,315$ ), felicidad (beta $=0,238$ ), sobreconfianza (beta $=0,259$ ), celos-soledad (beta $=-0,233$ ), e impulsividad (beta $=-0,139$ ). Los coeficientes de regresión estandarizados beta indican que estas variables tienen cierto peso sobre la variable "autoestima". De acuerdo con esta afirmación, los porcentajes de varianza explicada (coeficientes de determinación ajustados) por cada una de las 5 variables predictoras fueron de adecuada magnitud $(35,2 \%, 41,4 \%, 43,6 \%, 47,8 \%, 49,1$ $\%)$. Cinco variables resultaron predictoras de "autoestima": bajo nivel de depresión, muchos sentimientos de felicidad, alta sobreconfianza, pocos sentimientos de celos-soledad y baja impulsividad. El poder explicativo de estas variables predictoras es medio, ya que estas variables explican el $49,1 \%$ de la varianza.

Del conjunto de las variables predictoras de autoconcepto (ver Tabla 3), 5 resultaron estadísticamente significativas: felicidad (beta $=0,305$ ), habilidades sociales apropiadas (beta $=0,345$ ), autoexigencia profesional (beta $=0,164$ ), dinamismo (beta $=0,104$ ) y depresión (beta $=-0,117)$. Los coeficientes de regresión estandarizados beta indican que estas variables tienen cierto peso sobre la variable "autoconcepto". De acuerdo con esta afirmación, los porcentajes de varianza explicada por cada una de las 5 variables predictoras fueron de magnitud adecuada $(39,2 \%, 51,3 \%, 54,9 \%, 55,6 \%, 56,2 \%)$. Cinco variables resultaron predictoras de "autoconcepto": alto nivel de sentimientos de felicidad, muchas habilidades sociales apropiadas, alta autoexigencia profesional, alto dinamismo y bajo nivel de depresión. El poder explicativo de estas variables predictoras de la variable criterio es medio-alto, ya que estas variables explican el $56,2 \%$ de la varianza.

\section{DISCUSIón}

Los resultados del análisis descriptivo evidencian que los adolescentes (chicos) tienen puntuaciones significativamente superiores en autoestima, asertividad inapropiada, sobreconfianza, desajuste emocional y tolerancia al estrés, mientras que las adolescentes (chicas) muestran superiores puntuaciones en cooperación, habilidades sociales apropiadas, adaptación a los cambios, disponibilidad hacia 
nuevas tareas, capacidad para trabajar en equipo, ansiedad y depresión. Por consiguiente, se ratifica parcialmente la hipótesis 1 ya que los chicos tuvieron puntuaciones significativamente más altas en autoestima, pero no se dieron diferencias en relación al autoconcepto entre ambos sexos. Esto apunta en la dirección de diversos estudios que han encontrado puntuaciones inferiores en la autoestima de las chicas (Chabrol et al. 2004; Gracia et al. 1999; Hagborg, 1993; Khanlou, 2004; Locker y Cropley, 2004; Robison et al. 2001; Walker, 2000), y también de los estudios que no han encontrado diferencias en el autoconcepto global durante la adolescencia (Garaigordobil et al. 2003). Tal vez las puntuaciones inferiores de la autoestima de las chicas puede deberse a que éstas experimentan mayores niveles de estrés durante la adolescencia, probablemente como un resultado de factores educacionales, produciéndose mayor distancia entre la autoimagen y la imagen ideal.

Además, los resultados obtenidos ratifican la hipótesis 2 ya que se confirma que las chicas tienen puntuaciones superiores en diversos tipos de conductas sociales positivas (cooperación, habilidades sociales apropiadas, capacidad para trabajar en equipo), frente a los chicos que muestran puntuaciones significativamente más altas en asertividad inapropiada y sobreconfianza. Estas puntuaciones significativamente superiores de las chicas en conducta social positiva confirman los estudios que han sugerido que las adolescentes tienen puntuaciones significativamente superiores en competencia social (Vordach, 2002), en habilidades sociales apropiadas (Méndez et al. 2002), en estrategias y conductas prosociales (Pakaslahti et al. 2002), así como en adaptación social (Clemente et al. 1998). Complementariamente, se confirma la hipótesis 3 ya que, en la misma dirección que otros estudios, los resultados sugieren que no existen diferencias entre chicos y chicas en sentimientos de felicidad (Mahon et al. 2005), mientras que si se confirma que las chicas tienen más síntomas depresivos (Chabrol et al. 2004; Locker y Cropley, 2004) y de ansiedad (Locker y Cropley, 2004).

Una explicación de algunos de estos resultados, como son el menor nivel de autoestima o el mayor nivel de depresión y ansiedad en las chicas, puede estar asociada a un mayor nivel de autocrítica de las mujeres en la evaluación de sí mismas, de su personalidad, 
asi como una mayor capacidad para identificar "lo que falta" en contraposición a los varones, con mayor capacidad de identificar "lo que tienen", lo que conduce a las mujeres a un mayor desarrollo de sentimientos ansioso-depresivos.

Por otro lado, los resultados de los análisis correlacionales sugieren que, con mayor probabilidad, los adolescentes de ambos sexos con altas puntuaciones en autoconcepto y autoestima son cooperativos, tienen sentimientos de felicidad, manifiestan alta tolerancia al estrés, alto nivel de inteligencia social, de integración social, de capacidad de trabajar en equipo, son autoexigentes, perseverantes, manifiestan baja asertividad inapropiada, baja impulsividad, experimentan pocos sentimientos de celos-soledad, tienen un bajo nivel de desajuste emocional, así como de ansiedad y depresión, es decir, son estables emocionalmente, sociables y responsables.

Estos resultados confirman la hipótesis 4, y apuntan en la misma dirección que otros estudios que han evidenciado que los adolescentes con alto autoconcepto (Calvo et al. 2001; Garaigordobil et al. 2003; Gutiérrez y Clemente, 1993; Hay et al. 1998) y alta autoestima (Rigby y Slee, 1993) tienen muchas conductas sociales positivas. También se confirman los resultados de las investigaciones que han mostrado que los adolescentes con alta autoestima (Deluty, 1981; Bijstra et al 1994; Lefevre y West, 1981; Lorr y More, 1980; Riggio et al. 1990) y alto autoconcepto (Garaigordobil et al. 2003; Russomano, 2000) disponen de habilidades sociales apropiadas, siendo asertivos; así como los trabajos que han encontrado que los adolescentes con alta autoestima tienen pocas conductas de timidez (Lawrence y Bennett, 1992), baja impulsividad (Heaven, 1991), pocos celos (Buunk, 1997), pocos sentimientos de soledad (Brage et al. 1993; Haines et al. 1993; Moroi, 1987), muchos sentimientos de felicidad (Neto, 2001; Myers y Diener, 1995), y alto nivel de constancia-persistencia (Di Paula y Capmbell, 2002; Mc Farlin, Baumeister y Blascovich, 1984). Además, nuestro estudio ratifica los resultados de las investigaciones que han hallado relaciones negativas entre autoconcepto y depresión (Alfeld y Sigelman, 1998; Fan y Fu, 2001; Garaigordobil et al. 2005; Yang, 2002), entre autoestima y depresión (Garaigordobil et al. 2005; Hoffmann, Baldwin y Cerbone, 2003; Valentine, 2001), así como entre autoestima y ansiedad (Fickova, 1999; Newbegin y Owens, 1996; Yang, 2002). 
Finalmente, en este estudio se identifican como variables predictoras de la autoestima y el autoconcepto, pocos sentimientos de depresión, muchos sentimientos de felicidad, habilidades sociales adecuadas y bajo nivel de impulsividad, confirmándose la hipótesis 5. Estos resultados ratifican los obtenidos en estudios que han concluido que bajo autoconcepto (Braithwaite, 2004; Dowd, 2002) y baja autoestima (Hong, 2002) son predictores de depresión; o los que han identificado una alta autoestima social como predictor de asertividad (Arbanas, 1997), un alto autoconcepto como predictor de adaptación social (Garaigordobil et al. 2003), o una alta autoestima como predictora de felicidad (Coopersmith, 1967; Cheng y Furnham, 2003).

Los resultados obtenidos en el estudio sugieren la existencia de conexiones significativas entre alto autoconcepto-autoestima, conducta social adecuada, estabilidad emocional y ausencia de síntomas psicopatológicos ansioso-depresivos. Esta información puede tener relevantes implicaciones teórico-prácticas para el ámbito de la intervención o tratamiento psicológico en la infancia y la adolescencia. No obstante, cabe destacar como limitación del estudio que siendo los datos de naturaleza correlacional, poco aportan sobre la relación causal que puede existir entre dichas variables, por lo que se sugiere el análisis del constructo autoconcepto-autoestima con una metodología de investigación experimental.

\section{REFERENCIAS BIBLIOGRÁFICAS}

Alfeld, L. C., y Sigelman, C. K. (1998). Sex differences in self-concept and symptoms of depression during the transition to college. Joumal of Youth and Adolescence, 27 (2), 219-244.

Amezcua, J. A., y Pichardo, C. (2000). Diferencias de género en autoconcepto en sujetos adolescentes. Anales de psicologia, 16, 207-214.

Arbanas, K. M. F. (1997). The effects of sociometric status on adolescents' social information processing and attributional style. Dissertation Abstract Intemational Section B: The Sciences and Engineering, 57(12-B), 7754.

Bauer, J. G. (1997). Changes in teachers' perceptions of students' self-concept as seen by a selected group of teachers who have 
had extensive training in cooperative learning: An exploratory study. Dissertation Abstracts International Section A: Humanities and Social Sciences. 57 (11-A), 4645.

Bijstra, J. O., Bosma, H. A., y Jackson, S. (1994). The relationship between social skills and psycho-social functioning in early adolescence. Personality and Individual Differences, 16 (5), 767776.

Brage, D., Meredith, W. y Woodward, J. (1993). Correlates of loneliness among Midwestern adolescents, Adolescence, 28 (111), 685-693.

Braithwaite ${ }_{1}$ K. (2004). Predictors of depressive symptomatology among black college women. Dissertation Abstracts Intemational: Section $B$ : The Sciences and Engineering, 64 (12-B), 6322.

Buunk, B. P. (1997). Personality, birth order and attachment styles as related to various types of jealousy. Personality and Individual Differences, 23 (6), 997-1006.

Calvo, A. J., González, R., y Martorell, M. C. (2001) Variables relacionadas con la conducta prosocial en la infancia y adolescencia: personalidad, autoconcepto y género. Infancia y Aprendizaje, 24, 95-111.

Cardenal, V., y Fierro, A. (2003). Componentes y correlatos del autoconcepto en la escala de Piers-Harris. Estudios de Psicología, 24, 101-111.

Chabrol, H., Carlin, E., Michaud, C., Rey, A., Cassan, D., Juillot, M., Rousseau, A., Callahan, S. (2004). A study of the Rosenberg selfesteem scale in a sample of high-school students. Neuropsychiatrie del'Enfance et del'Adolescence, 52 (8), 533-536.

Cheng, H., y Furnham, A. (2003). Personality, self-esteem, and demographic predictions of happiness and depression. Personality and Individual Differences, 34 (6), 921-942.

Clemente, A., Albiña, P., y Domenech, F. (1998). Análisis de las diferencias entre sexos, en socialización y adaptación escolar en alumnos de los dos primeros cursos de secundaria. Psicologia Educativa. Revista de los Psicólogos de la Educación, 4 (2), 157-166.

Coopersmith, S. (1967). The antecedents of self-esteem. San Francisco: Freeman.

Corral, S., Pamos, A., Pereña, J., y Seisdedos, N. (2002). TPT. Test de Personalidad de TEA. Madrid: TEA. 
Deluty, R. H. (1981). Adaptiveness of aggressive, assertive, and submissive behavior for children. Journal of Clinical Child Psychology, 10 (3), 155-158.

Derogatis, L. R. (2002). SCL-90-R. Cuestionario de 90 sintomas revisado. Madrid: TEA. (trabajo original publicado en 1983).

Di Paula, A. \& Campbell, J. D. (2002). Self-esteem and persistence in the face of failure. Journal of Personality and Social Psychology, 83 (3), 711-724.

Dowd, S. A. (2002). Internalizing symptoms in adolescents: Assessment and relationship to self-concept. Dissertation Abstract International Section B: The Sciences and Engineering, 62 (8-B), 3796.

Eskin, M. (2003). Self-reported assertiveness in Swedish and Turkish adolescents: A cross-cultural comparison. Scandinavian Journal of Psychology, 44 (1), 7-12.

Fan, F., y Fu, J. (2001). Self-concept and mental health of college students. Chinese Mental Health Joumal, 15, 76-77.

Fickova, E. (1999). Personality dimensions and self-esteem indicators relationships. Studia Psychologica, 41, 323-328.

Garcia, F., y Musitu, G (1999). AF-5. Autoconcepto Forma 5. Madrid:TEA. Garaigordobil, M. (1996). Evaluación de una intervención psicoeducativa en sus efectos sobre la conducta prosocial y la creatividad. Madrid. Ministerio de Educación y Ciencia. CIDE. Colección Investigación $n^{2} 127$. Primer Premio Nacional de Investigación Educativa 1994.

Garaigordobil, M. (1999a). Assessment of a cooperative-creative program on of assertive behavior and self-concept. Spanish Joumal of Psychology, 2 (1), 3-10.

Garaigordobil, M. (1999b). Diseño y evaluación de un programa de intervención psicoeducativa para la educación en derechos humanos durante la adolescencia. En Premios Nacionales de Investigación Educativa 1998 (pp. 115-150). Madrid: Ministerio de Educación y Cultura. CIDE. Colección Investigación $n^{2} 142$. Tercer Premio Nacional de Investigación Educativa 1998.

Garaigordobil, M. (2002). Assessment of an intervention on social behavior, intragroup relations, self-concept and prejudiced cognitions during adolescence. International Journal of Psychology and Psychological Therapy, 2(1), 1-22. 
Garaigordobil, M. (2005). Diseño y evaluación de un programa de intervención socioemocional para promover la conducta prosocial y prevenir la violencia. Madrid: Ministerio de Educación y Ciencia. CIDE. Colección Investigación $n^{2} 160$. Primer Premio Nacional de Investigación Educativa 2003.

Garaigordobil, M. (en prensa). LAEA. Listado de adjetivos para la evaluación del autoconcepto en adolescentes y adultos. Madrid: Pirámide.

Garaigordobil, M., Cruz, S., y Pérez, J. I. (2003). Análisis correlacional y predictivo del autoconcepto con otros factores conductuales, cognitivos y emocionales de la personalidad durante la adolescencia. Estudios de Psicologla, 24, 113-134.

Garaigordobil, M., Durá, A., y Pérez, J. I. (2005). Psychopathological symptoms, behavioural problems, and self-concept/self-esteem: A study with adolescents from 14 to 17 years. Annuary of Clinical and Health Psychology, 1, 53-63.

Garaigordobil, M., y Echebarria, A. (1995). Assessment of a peer-helping game program on children's development. Journal of Research in Childhood Education, 10(1), 63-69.

Gracia, M., Marco, M., Fernández, M., y Juan, J. (1999). Autoconcepto físico, modelo estético e imagen corporal en una muestra de adolescentes. Psiquis, 20 (1), 27-38.

Gutiérrez, M., y Clemente, A. (1993). Autoconcepto y conducta prosocial en la adolescencia temprana: bases para la intervención. Revista de Psicología de la Educación, 4 (11), 39-48.

Hagborg, W. J. (1993). The Rosenberg Self-Esteem Scale and Harter's Self-Perception Profile for adolescents: A concurrent validity study. Psychology in the Schools, 30 (2), 132-136.

Haines, D. A., Scalise, J. J., y Ginter, E. J. (1993). Relationship of loneliness and its affective elements to self-esteem. Psychological Reports, 73 (2), 479-482.

Hay, I., Ashman, A. F., y Van Kraayenoord, C. E. (1998). Educational characteristics of students with high or low self-concept. Psychology in the Schools, 35 (4), 391-400.

Heaven, P. C. (1991). Personality correlates of functional and dysfunctional impulsiveness. Personality and Individual Differences, 12 (11), 1213-1217. 
Hills, P., y Argyle, M. (2002). The Oxford Happiness Questionnaire: a compact scale for the measurement of psychological well-being. Personality and Individual Differences, 33, 1073-1082.

Hoffmann, J. P., Baldwin, S. A., y Cerbone, F. G (2003). Onset of major depressive disorder among adolescent. Journal of the American Academy of Child and Adolescent Psychiatry, 42 (2), 217-224.

Hong, Z.R. (2002). An investigation of self-esteem and school achievement of Taiwanese secondary students. Dissertation Abstracts International Section A: Humanities and Social Sciences, $63(1-A), 82$.

Khanlou, N. (2004). Influences on adolescent self-esteem in multicultural canadian secondary schools. Public Health Nursing, 21 (5), 404411.

Klomsten, A.T., Skaalvik, E. M., y Espnes, G. A (2004). Physical selfconcept and sports: Do gender differences still exist? Sex-Roles, 50 (1-2), 119-127.

Lameiras, M., y Rodríguez, Y. (2003). Age and sex differences in selfesteem among spanish adolescentes. Psychological Reports, 93, 876-878.

Lawrence, B., y Bennett, S. (1992). Shyness and education: The relationship between shyness, social class and personality variables in adolescents. British Joumal of Educational Psychology, 62 (2), 257-263.

Lefevre, E. R., y West, M. L. (1981). Assertiveness: Correlations with self-esteem locus of control, interpersonal anxiety, fear of disapproval, and depression. Psychiatric Journal of the University of Ottawa, 6(4), 247-251.

Locker, J., y Cropley, M. (2004). Anxiety, depression and selfesteem in secondary school children: an investigation into the impact of standard assessment tests (SATS) and other important school examinations. School Psychology International, 25 (3), 333-345.

Lorr, M., y More, W. W. (1980). Four dimensions of assertiveness. Multivariate Behavioral Research, 15 (2), 127-138.

Mahon, N. E., Yarcheski, A., y Yarcheski, T. J. (2005). Happiness as Related to Gender and Health in Early Adolescents. Clinical Nursing Research, 14 (2), 175-190. 
McCarthy, J. D., y Hoge, D. R. (1982). Analysis of age effects in longitudinal study of adolescent self-esteem. Developmental Psychology, 18, 372-379.

Mc Farlin, D. B., Baumeister, R. F., y Blascovich, J. (1984). On knowing when to quit: Task failure, self-esteem, advice, and nonproductive persistence. Journal of Personality, 52 (2), 138-155.

Matud, P., Ibáñez, I., Marrero, R., y Carballeira, M. (2003). Diferencias en autoestima en función del género [Gender differences in selfesteem]. Análisis y Modificación de Conducta, 29, 51-78.

Méndez, X., Hidalgo, M. D., e Ingles, C. (2002). The Matson Evaluation

of Social Skills with youngsters: psychometric properties of the spanish translation in the adolescent population. European Joumal of Psychological Assessment, 26 (2), 137-144.

Moroi, K. (1987). Loneliness and self-consciousness in high-school students. Japanese Journal of Psychology, 56 (4), 237-240.

Myers, D. G, y Diener, E. (1995). Who is happy?. Psychological Science, 6(1), 10-19.

Neto, F. (2001). Personality predictors of happiness. Psychological Reports, 88 (3), 817-824.

Newbegin, I., y Owens, A. (1996). Self-esteem and anxiety in secondary school achievement. Journal of Social Behavior and Personality, 11, 521-530.

Pakaslahti, L., Karjalainen, A., Keltikangas, L. (2002). Relationships between adolescent prosocial problem-solving strategies, prosocial behaviour, and social acceptance. International Joumal of Behavioral Development, 26 (2), 137-144.

Pastor, Y., Balaguer, I., y García-Merita, M. L. (2003). El autoconcepto y la autoestima en la adolescencia media: análisis diferencial por curso y género. Revista de Psicología Social, 18 (2), 141-159.

Rigby, K., Cox, I., y Black, G (1997). Cooperativeness and BullyNictim

Problem among australian schoolchildren. The Journal of Social Psychology, 137 (3), 357-368.

Rigby, K., y Slee, P.T. (1993). Dimensions of interpersonal relation among Australian children and implications for psychological wellbeing. Journal of Social Psychology, 133 (1), 33-42.

Riggio, R. E., Throckmorton, B., y De Paola, S. (1990). Social skills and self-esteem. Personality and Individual Differences, 11 (8), 799-804. 
Robison, A. P., Kehle, T.J., Bray, M. A., Jenson, W. R., Clark, E., Lawless, K. A. (2001). Self-esteem, gender-role perception, genderrole orientation and attributional style as a function of academic competence: Smart girls are different, but a boy is a boy is a boy. Canadian Journal of School Psychology, 17 (1), 47-64.

Rosenberg, M. (1965). Society and the adolescent self-image. Princeton, NJ: Princeton University Press.

Russomano, L. (2000). Achievement, locus of control, self-concept, social problem-solving training and the acquisition of prosocial skills in children. Dissertation Abstracts International Section A: Humanities and Social Sciences, 61 (2-A), 485.

Silber, E., y Tippett, J. (1965). Self-esteem: clinical assessment and measurement validation. Psychological Reports, 16, 1017-1071.

Takakura, M., y Sakihara, S. (2001). Psychosocial correlates of depressive symptoms among Japanese high school students. Journal of Adolescent Health, 28, 82

Trianes, M. V., Blanca, M. J., Muñoz, A., García, B., Cardelle-Elawar M., e Infante L. (2002). Relaciones entre evaluadores de la competencia social en preadolescentes: Profesores, iguales y autoinformes. Anales de Psicología, 18 (2), 197-214.

Valentine, I. S. (2001). The relationship between depression, selfesteem, trauma, and psychopathy in understanding conduct disordered adolescents. Dissertation Abstracts International Section $B$ : The Sciences and Engineering, 61 (10-B), 5585.

Veenman, S., Kenter, B., y Post, K. (2000). Cooperative learning in Dutch primary classrooms. Educational Studies. 26 (3), 281-302. Vordach, A.M. (2002). The relationship between emotional competence and social competence among early adolescents. Dissertation Abstracts International: Section B: The Sciences and Engineering, 63 (3-B), 1578.

Walker, H. R. (2000) An analysis of the relationship of human sexuality knowledge, self-esteem, and body image to sexual satisfaction in college and university students. Dissertation Abstract Intemational: Section B: The Sciences and Engineering, 60 (9-B), 4560.

Yang, D. (2002). Depression, anxiety, and self-concept of middle school students. Chinese Mental Health Journal, 16, 633-635. 